\title{
Proposed Precoder for the Secondary Transmitter in the Cognitive MIMO Radio Network
}

\author{
Hoai Trung Tran \\ University of Transport and Communications \\ Hanoi, Vietnam
}

\begin{abstract}
Currently, cognitive radio systems (CRSs) are of great interest to improve the efficiency of using the available spectrum. The spectrum sharing technique to share the spectrum between a primary transmitter (PT) and a secondary transmitter (ST) is influential in CRSs. However, when reusing the PT spectrum, the ST transmits energy and decoded data to the secondary users (SUs), and providing power to the energy receivers (ERs) also interferes with the primary users (PUs). One method used to increase the power delivered to the ERs, limit the interference to the PUs and increase the total channel capacity to the SUs is to use the weighted minimum mean squared error (WMMSE) method. This method combines increasing the channel capacity to the SUs and minimizing the error for the transmission channels. It will not focus on solving error reduction but rather on increasing the channel capacity through the precoder design at the STs. The proposed algorithm combines using the eigenvectors of the known channel matrix at the ST to generate beams; the distributed power value for each beam is calculated based on the Lagrangian operator combined with the Karush-Kuhn-Tucker (KKT) conditions to maximize capacity. This new method allows increased capacity compared to systems using WMMSE by other methods such as weighted sum rate (WSR), harmonic mean rate (HMR), or proportional fairness (PF) in different situations such as increasing the number of SUs, ERs, or PUs or limiting interference to given PUs..
\end{abstract}

\section{General Terms}

Algorithms in signal processing

\section{Keywords}

Cognitive Radio Systems, primary transmitter, primary users, secondary transmitter, seconday users, energy receivers, transmit precoders

\section{INTRODUCTION}

Cognitive radio systems (CRSs) are considered a solution for making the best use of spectrum resources. It is defined as a network that can observe, act, learn and optimize for quality enhancement [1]. The next-generation wireless networks require intelligent and reconfigurable radio waves (popularly known as cognitive radio (CR)). They can interact with a constantly changing environment to realize real-time spectrum and take advantage of any available spectrum usage opportunities [2].

Also, according to [3], cognitive radio uses heterogeneous CRS and spectrum sharing CRS. Radio Access Networks (RANs) can use fixed frequency bands in heterogeneous CRS cases, and in the second case, RANs can use the same frequency band. CRS has two main techniques; one is full CRS, where the system estimates all parameters, one node can know the parameters for another node. Another technique is spectrum sensing that is related closely to the spectrum sharing case. A secondary node knows the frequency channels in spectrum sensing use and knows which frequency has been allocated to the primary node. A challenge in cognitive radio is that the secondary users need to reasonably separate the presence of primary users in the licensed spectrum and leave the band as quickly as possible when the primary radio requires it to ensure that it does not affect primary users. The technique is called spectrum sensing. Spectrum sensing and its estimation are the first steps to implement for CRS systems [4].

In the context of cognitive radio (CR), a licensed universal user is called a primary user (PU), and an unlicensed user is referred to as a secondary user (SU) or a CR node (both terms will be used interchangeably). Therefore, the SU must have the opportunity to access the spectrum holes while keeping the noise on the PU receiver at zero or below a given level (spectrum leasing). Cognitive radio has been considered as a potential means of improving spectral efficiency by allowing the user (SU) to share the spectrum initially allocated to the primary user (PU), as long as the total interference at the primary receiver is below the acceptable level [11]

Cognitive radio networks can also be thought of as consisting of a cognitive base station or secondary transmitter (ST) and multiple cognitive users or secondary users (SUs), in addition to the presence of eavesdroppers (EAs). The ST first detects a spectral hole through the spectrum sensor in the cognitive radio network and then communicates with the SUs through the spectral detection hole [12]. This paper also assumes that a cognitive radio network with one $\mathrm{ST}$ and one $\mathrm{SU}$ is opportunistically authorized access to the main network consisting of a primary transmitter (PT) and a user primary user (PU). Specifically, if the PT and the PU communicate over the licensed spectrum, the ST and the SU are not allowed to transmit. If the licensed spectrum is found to be inactive (the PT and the PU are not using, i.e., the spectrum), the ST and the SU can reuse the idle spectrums (also known as spectral holes) for data transmission [12].

In summary, in the cognitive radio system components, there are always the primary transmitters (PTs) and the primary receivers (PUs), the secondary transmitters (STs), and the secondary receiver (SU), in addition to energy-harvesting users (potential eavesdroppers) (ERs or EAs). Thus, in the CRS systems, precoders are of interest to both the PT and the ST because they are sources of power and information transmission.

The article on precoder for the ST to maximize the total rate [6] is interested in designing a precoder for the PT and the ST to ensure the maximum speed of the primary link from 
the PT to the PU. Here, there is a constraint on the obtained energy of the SUs due to their energy harvesting. Based on the block coordinate descent (BCD) proposition, the paper presents the separation of the secrecy primary rate optimization into two convex functions for the precoder at the PT and the ST, using two more updated auxiliary parameters related to the channel matrices between the PT, the ET, and the PU, the EA. The article [7] is interested in the design from the ST to the SUs. There are constrained conditions for the transmit power at the ST, noise limit from the ST to the PU, and the energy harvesting limit of the ERs. The article [9] deals with power division at the ST in which part of the power is used to transmit the confidential message to the PU, the remaining power, using energy harvesting technique, is transmitted to the SUs. Corresponding to the two transmission directions, they have the forwarding beamforming matrix for the PUs and the cognitive beamforming matrix for the SUs. The goal is also to maximize the capacity of the SUs from the ST using a cognitive beamforming matrix. At the same time, there are constraints on the capacity generated by forwarding beamforming matrix to the SUs, the signal generated by forwarding beamforming matrix to the PUs or the ERs must be higher or smaller than the cognitive beamforming matrix generated respectively, the constraints of forwarding and cognitive beamforming matrix themselves, as well as these two matrices, must be greater than 0 . The article [10] focuses on understanding the ST as a relay for the SUs. Here, the precoder of the ST and the two SUs are optimized to maximize the total speed to two SUs from the ST, adding some constraints on limiting interference to the PU from the two SUs and the ST, the minimum speed from the ST to two SUs, or the maximum power of the ST.

Article [6] is concerned with the secrecy rate from the PT to the PU. It is taking into account the influence of the ST. In addition, it deals with the energy from the PT to the ERs with support along with the ST. In the meanwhile, articles [7] ] to [10] are interested in the speed from the ST to the SUs. Articles [7-10] take into account the constraint of interference from ST to PU, in which [7] is concerned with the energy supplied from the ST to the ERs and [8] takes into account the error of radio channel state information. The article [9] has added that the limited energy constraint is granted from the ST to the eavesdroppers (EAs). Mainly, article [10] gives many constraints, including the PT's noise to the ST and the SUs, noise from the SUs to the PU. Article [10] has highlighted the relay role of the ST. Articles [6] and [8] are interested in a function that maximizes the intermediate parameter. This parameter is related to the precoder of PT and ST, which is quite similar to the WMMSE function that also has this parameter in paper [7]. Articles [6-9] are probably related to the Lagrange operator and the function to be optimized and the constraints, combined with the KKT condition. Remarkably, the article [10] mentions using eigenvectors of the channel matrix used as precoder of the SUs related to the ST, while the power distribution values for the eigenvectors can be calculated according to the Lagrange operator.

A proposed method is given that using the eigenvectors of the channel matrix as precoders at the ST, the power values are calculated based on the general Lagrangian operator. This method is performed when the interference from the STs to the PUs is small enough and the energy suggested at the ERs is large enough. This method gives higher capacity in using WMMSE with various methods such as WSR, HMR, and PR [7].

\section{CHANNEL MODEL}

I explore the optimal precoder design in a standard MuMIMO CR simultaneous wireless information and power transfer (SWIPT) network. An ST supports multiple secondary SUs, and multiple ER users all have multiple antennas using spectrum license, specified for the PU users.

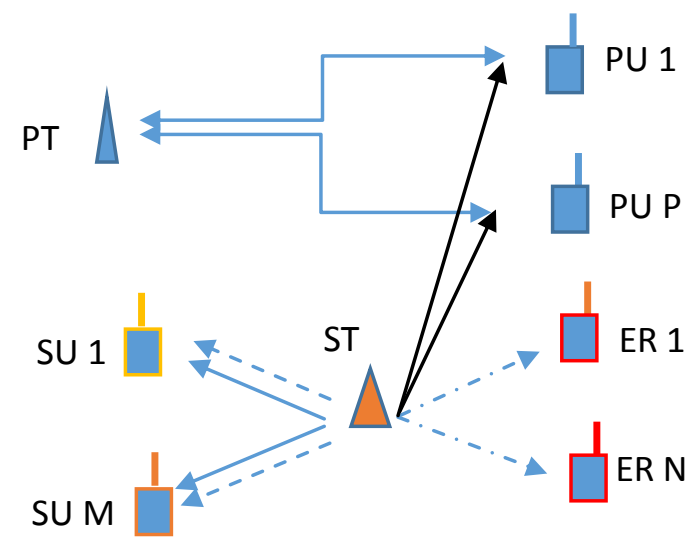

Fig. 1: The MuMIMO CR SWIPT network

Signals received by the mobile SU:

$$
\mathbf{y}=\mathbf{H F x}+\mathbf{n}
$$

With $\mathbf{H}=\left[\left\{\mathbf{H}_{\mathrm{k}}^{\mathrm{T}}\right\}_{\mathrm{k}=1}^{\mathrm{M}}\right]$ and $\mathbf{F}=\left[\left\{\mathbf{F}_{\mathrm{k}}\right\}_{\mathrm{k}=1}^{\mathrm{M}}\right]$.

Where $\mathbf{H}, \mathbf{H}_{\mathrm{k}}$ have sizes of MExG, ExG and $\mathbf{F}, \mathbf{F}_{\mathrm{k}}$ have sizes of FxMG , FxG, in which E, G are number of antennas of ST and SU.

The goal is that I optimize the function that maximizes the total rate from the ST to the SUs where the rate from the ST to the $\mathrm{k}$ th $\mathrm{SU}$ is:

$$
\mathrm{R}_{\mathrm{k}}=\log \operatorname{det}\left(\mathbf{F}_{\mathrm{k}}^{\mathrm{H}} \mathbf{H}_{\mathrm{k}}^{\mathrm{H}} \mathbf{R}_{\mathrm{n}, \mathrm{k}}^{-1} \mathbf{H}_{\mathrm{k}} \mathbf{F}_{\mathrm{k}}+\mathbf{I}_{\mathrm{M}}\right)
$$

Sum rate will be:

$$
\max _{\mathbf{F}} \sum_{\mathrm{k}=1}^{\mathrm{M}} \mathrm{R}_{\mathrm{k}}
$$

With the constrains:

$\operatorname{Tr}\left(\mathbf{F}^{\mathrm{H}} \mathbf{F}\right) \leq \mathrm{P}_{\mathrm{T}} \quad$ (the ST's power must not exceed the allowable threshold).

$\operatorname{Tr}\left(\mathbf{F}^{\mathrm{H}} \mathbf{G}_{\mathrm{i}}^{\mathrm{H}} \mathbf{G}_{\mathrm{i}} \mathbf{F}\right) \geq \mathrm{E}_{\mathrm{th}, \mathrm{i}}, \mathrm{i}=1: \mathrm{N}$ (The power from the ST transmitted to the ERs using harvesting energy must be large enough; $\mathbf{G}_{\mathrm{i}}$ is the channel matrix from the ST to the ith PU).

$\operatorname{Tr}\left(\mathbf{F}^{\mathrm{H}} \mathbf{T}_{\mathrm{j}}^{\mathrm{H}} \mathbf{T}_{\mathrm{j}} \mathbf{F}\right) \leq \mathrm{I}_{\mathrm{th}, \mathrm{j}}, \mathrm{i}=1: \mathrm{P} \quad$ (Interference power from the ST to P PUs must be small enough; $\mathbf{T}_{\mathrm{j}}$ is the channel matrix from the ST to the $\mathrm{j}$ th $\mathrm{PU}$ ).

The article [7] uses the WMMSE standard to maximize the capacity of equation (3) and ensure minimum error. Equation (1) is rewritten as: 


$$
\inf _{\gamma, \overline{\mathbf{F}},\left\{\mathbf{W}_{\mathrm{k}}, \mathbf{L}_{\mathrm{k}}, \forall \mathrm{k}\right\}} \sum_{\mathrm{k}=1}^{\mathrm{M}}\left\{\operatorname{Tr}\left(\mathbf{W}_{\mathrm{k}} \mathbf{C}_{\mathrm{k}}\right)+\mathrm{e}_{\mathrm{k}}\left(\mathbf{W}_{\mathrm{k}}\right)\right\}
$$

With constraints:

$$
\begin{aligned}
& \operatorname{Tr}\left(\mathbf{F}^{\mathrm{H}} \mathbf{F}\right) \leq \gamma^{-2} \mathrm{P}_{\mathrm{T}} \\
& \operatorname{Tr}\left(\mathbf{F}^{\mathrm{H}} \mathbf{G}_{\mathrm{i}}^{\mathrm{H}} \mathbf{G}_{\mathrm{i}} \mathbf{F}\right) \geq \gamma^{-2} \mathrm{E}_{\text {th }, \mathrm{i}}, \mathrm{i}=1: \mathrm{N} \\
& \operatorname{Tr}\left(\mathbf{F}^{\mathrm{H}} \mathbf{T}_{\mathrm{j}}^{\mathrm{H}} \mathbf{T}_{\mathrm{j}} \mathbf{F}\right) \leq \gamma^{-2} \mathrm{I}_{\mathrm{th}, \mathrm{j}}, \mathrm{j}=1: \mathrm{P}
\end{aligned}
$$

To maximize this function, I introduce the Lagrange operator:

$$
\begin{aligned}
& \mathrm{L}_{\mathrm{p}}=\operatorname{Tr}(\mathbf{W C})+\mathrm{e}(\mathbf{W})+v\left(\operatorname{Tr}\left(\overline{\mathbf{F}} \overline{\mathbf{F}}^{\mathrm{H}}\right)-\gamma^{-2} \mathrm{P}_{\mathrm{T}}\right) \\
& -\sum_{\mathrm{i}=1}^{\mathrm{N}} \lambda_{\mathrm{i}}\left(\operatorname{Tr}\left(\overline{\mathbf{F}}^{\mathrm{H}} \mathbf{G}_{\mathrm{i}}^{\mathrm{H}} \mathbf{G}_{\mathrm{i}} \overline{\mathbf{F}}-\gamma^{-2} \mathrm{I}_{\mathrm{th}, \mathrm{j}}\right)\right) \\
& +\sum_{\mathrm{j}=1}^{\mathrm{M}} \mu_{\mathrm{j}}\left(\operatorname{Tr}\left(\mathbf{T}_{\mathrm{j}} \mathbf{S} \mathbf{T}_{\mathrm{j}}^{\mathrm{H}}-\gamma^{-2} \mathrm{I}_{\mathrm{th}, \mathrm{j}}\right)\right) \\
& \mathbf{C}=\operatorname{blkdiag}\left(\mathbf{C}_{1}, \ldots, \mathbf{C}_{\mathrm{M}}\right) \\
& =\mathbf{L}\left(\mathbf{H} \overline{\mathbf{F}} \overline{\mathbf{F}}^{\mathrm{H}} \mathbf{H}^{\mathrm{H}}+\gamma^{-2} \sigma_{\mathrm{n}}^{2} \mathbf{I}_{\mathrm{ME}}\right) \mathbf{L}^{\mathrm{H}} \\
& -\mathbf{L H} \overline{\mathbf{F}}-\overline{\mathbf{F}}^{\mathrm{H}} \mathbf{H}^{\mathrm{H}} \mathbf{L}^{\mathrm{H}}+\mathbf{I}_{\mathrm{ME}}
\end{aligned}
$$$$
\text { Here, } \quad=\mathbf{L}\left(\mathbf{H} \overline{\mathbf{F}} \overline{\mathbf{F}}^{\mathrm{H}} \mathbf{H}^{\mathrm{H}}+\gamma^{-2} \sigma_{\mathrm{n}}^{2} \mathbf{I}_{\mathrm{ME}}\right) \mathbf{L}^{\mathrm{H}}
$$

Necessary conditions for the KKT standards:

1. $\mathbf{L}_{\mathrm{k}}\left(\mathbf{H}_{\mathrm{k}} \overline{\mathbf{F}}_{\mathrm{k}} \overline{\mathbf{F}}_{\mathrm{k}}^{\mathrm{H}} \mathbf{H}_{\mathrm{k}}^{\mathrm{H}}+\gamma^{-2} \mathrm{R}_{\mathrm{n}, \mathrm{k}}\right)=\overline{\mathbf{F}}_{\mathrm{k}}^{\mathrm{H}} \mathbf{H}_{\mathrm{k}}^{\mathrm{H}}, \forall \mathrm{k}$

$\Rightarrow$ Optimum receiver:

$$
\mathbf{L}_{\mathrm{k}}=\overline{\mathbf{F}}_{\mathrm{k}}^{\mathrm{H}} \mathbf{H}_{\mathrm{k}}^{\mathrm{H}}\left(\mathbf{H}_{\mathrm{k}} \overline{\mathbf{F}}_{\mathrm{k}} \overline{\mathbf{F}}_{\mathrm{k}}^{\mathrm{H}} \mathbf{H}_{\mathrm{k}}^{\mathrm{H}}+\gamma^{-2} \mathbf{R}_{\mathrm{n}, \mathrm{k}}\right)^{-1}, \forall \mathrm{k}
$$

2. $\mathbf{C}_{\mathrm{k}}^{\mathrm{T}}-\boldsymbol{\Gamma}_{\mathrm{k}}\left(\mathbf{W}_{\mathrm{k}}\right)=\mathbf{0}, \forall \mathrm{k}$

$\Rightarrow$ Optimum Iight matrix $\mathbf{W}_{\mathrm{k}}=\nabla_{\eta \mathrm{k}}\left(\mathbf{C}_{\mathrm{L}, \mathrm{k}}^{\mathrm{T}}\right), \forall \mathrm{k}$ where

$$
\mathbf{C}_{\mathrm{L}, \mathrm{k}}=\left(\gamma^{2} \overline{\mathbf{F}}_{\mathrm{k}}^{\mathrm{H}} \mathbf{H}_{\mathrm{k}}^{\mathrm{H}} \mathbf{R}_{\mathrm{n}, \mathrm{k}}^{-1} \mathbf{H}_{\mathrm{k}} \overline{\mathbf{F}}_{\mathrm{k}}+\mathbf{I}\right)^{-1}, \forall \mathrm{k}
$$

For example, $\quad \mathbf{W}_{\mathrm{k}}=\alpha_{\mathrm{k}} \mathbf{C}_{\mathrm{L}, \mathrm{k}}^{-1}, \forall \mathrm{k}$ with WSR (Weighted Sum Rate), $\mathbf{W}_{\mathrm{k}}=\left(-\left(\log \operatorname{det}\left(\mathbf{C}_{\mathrm{L}, \mathrm{k}}\right)\right) \mathbf{C}_{\mathrm{L}, \mathrm{k}}^{-1}\right)^{-1}, \forall \mathrm{k}$ with HMR (Harmonic Mean Rate) and $\mathbf{W}_{\mathrm{k}}=\left(\left(\log \operatorname{det}\left(\mathbf{C}_{\mathrm{L}, \mathrm{k}}\right)\right)^{2} \mathbf{C}_{\mathrm{L}, \mathrm{k}}^{-1}\right)^{-1}, \forall \mathrm{k}$ with $\quad$ PF $\quad$ (Proprtional Fairness).

3. $v\left(\operatorname{Tr}\left(\overline{\mathbf{F}} \overline{\mathbf{F}}^{\mathrm{H}}\right)-\gamma^{-2} \mathrm{P}_{\mathrm{T}}\right)=0$;

$\mu_{\mathrm{j}}\left(\operatorname{Tr}\left(\mathbf{T}_{\mathrm{j}} \mathbf{S} \mathbf{T}_{\mathrm{j}}^{\mathrm{H}}-\gamma^{-2} \mathbf{I}_{\mathrm{th}, \mathrm{j}}\right)\right)=0$

Therefore, I have:

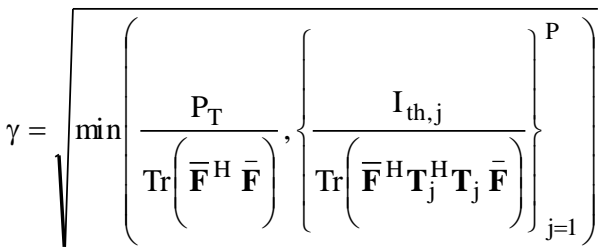

Algorithm:

1. The channel capacity setup

2. Given energy values $\left\{\mathrm{E}_{\mathrm{th}, 1}, \ldots, \mathrm{E}_{\mathrm{th}, \mathrm{N}}\right\}$

3. Creating $\mathrm{N}_{\mathrm{G}}$ random initial precoders: $\left\{\overline{\mathbf{F}}^{(1)}, \ldots, \overline{\mathbf{F}}^{\left(\mathrm{N}_{\mathrm{G}}\right)}\right\}$

4. For $i_{p}=1: N_{G}$

Initialization $\overline{\mathbf{F}}=\overline{\mathbf{F}}^{\left(\mathrm{i}_{\mathrm{p}}\right)}$, calculate $\gamma(8)$

Repeat

Calculate $\mathbf{L}, \mathbf{W}$ from (6), (7) correspondingly

Find $\overline{\mathbf{F}}^{*}=\mathbf{K}^{-1} \mathbf{H}^{\mathrm{H}} \mathbf{L}^{\mathrm{H}} \mathbf{W}^{\mathrm{H}}$

Continue to calculate $\gamma(8)$

Until convergence

Save $\mathbf{F}^{\left(\mathrm{i}_{\mathrm{p}}\right)}=\gamma \overline{\mathbf{F}}$

End for

Select the best one from $\left[\left\{\mathbf{F}^{\left(i_{p}\right)}\right\}\right]_{i_{p}=}^{N_{g}}$

Here, I should take care of the need-to-know usage of $\mathbf{H}_{\mathrm{k}}, \mathbf{R}_{\mathrm{n}, \mathrm{k}}$ at ST. Also, when creating $\mathbf{F}^{\left(\mathrm{i}_{\mathrm{p}}\right)}$ where $\mathrm{i}_{\mathrm{p}}=1: \mathrm{N}_{\mathrm{G}}$, then updating $\mathbf{F}^{\left(\mathrm{i}_{\mathrm{p}}\right)}$ again through $\mathbf{L}, \mathbf{W}, \mathbf{F}$, it seems random and not necessarily optimal $\mathbf{F}$.

\section{PROPOSED METHOD}

Another solution to increase the total speed is to maximize (2) so as not through WMMSE directly. This method combines capacity increase and error reduction, so it is not necessarily the proper method for optimizing the total capacity from the ST to the SUs.

With (2), I can infer the following: $\boldsymbol{\Sigma}_{\mathrm{k}}=\mathbf{H}_{\mathrm{k}}^{\mathrm{H}} \mathbf{R}_{\mathrm{n}, \mathrm{k}}^{-1} \mathbf{H}_{\mathrm{k}}=\mathbf{U}_{\mathrm{M}} \boldsymbol{\Lambda}_{\mathrm{M}} \mathbf{U}_{\mathrm{M}}^{\mathrm{H}}$, where $\mathbf{U}_{\mathrm{M}}, \boldsymbol{\Lambda}_{\mathrm{M}}$ are the eigenvectors and eigenvalues matrices of $\boldsymbol{\Sigma}_{\mathrm{k}}$. $\boldsymbol{\Lambda}_{\mathrm{M}}=\operatorname{diag}\left\{\sigma_{\mathrm{M}, 1}, \ldots, \sigma_{\mathrm{M}, \mathrm{M}}\right\}$ is a diagonal matrix with $\sigma_{\mathrm{M}, 1}, \mathrm{i}=1: \mathrm{M}$ are arranged in decreasing order.

If I use the singular value decomposition (SVD) for $\mathbf{F}_{\mathrm{k}}=\mathbf{U}_{\mathrm{k}} \boldsymbol{\Lambda}_{\mathrm{k}} \mathbf{V}_{\mathrm{k}}^{\mathrm{H}}$. When I substitute $\mathbf{F}_{\mathrm{k}}$ in (2), I will miss $\mathbf{V}_{\mathrm{k}}$ . The effective formula of $\mathbf{F}_{\mathrm{k}}=\mathbf{U}_{\mathrm{k}} \boldsymbol{\Lambda}_{\mathrm{k}}$. If given $\mathbf{U}_{\mathrm{k}}=\mathbf{U}_{\mathrm{M}}$, (2) becomes a power distribution problem:

$$
\mathrm{R}_{\mathrm{k}}=\sum_{\mathrm{k}=1}^{\mathrm{M}} \log \left(1+\sigma_{\mathrm{M}, \mathrm{k}} \mathrm{x}_{\mathrm{k}}\right)
$$

Where $\quad \mathbf{x}=\left[\mathrm{x}_{1}, \mathrm{x}_{2}, \ldots, \mathrm{x}_{\mathrm{M}}\right]=\left[\lambda_{\mathrm{k}, 1}^{2}, \lambda_{\mathrm{k}, 2}^{2}, \ldots, \lambda_{\mathrm{k}, \mathrm{M}}^{2}\right]$ is vector containg the squared power distribution coefficients of $\boldsymbol{\Lambda}_{\mathrm{k}}$. Constraints:

$$
\sum_{\mathrm{k}=1}^{\mathrm{M}} \mathrm{x}_{\mathrm{k}} \leq \gamma^{-2} \mathrm{P}_{\mathrm{T}}
$$




$$
\begin{gathered}
\sum_{\mathrm{k}=1}^{\mathrm{M}} \mathrm{x}_{\mathrm{k}} \mathrm{g}_{\mathrm{k}, \mathrm{i}} \geq \gamma^{-2} \mathrm{E}_{\mathrm{th}, \mathrm{i}} \text { inactive } \\
\sum_{\mathrm{k}=1}^{\mathrm{M}} \mathrm{x}_{\mathrm{k}} \mathrm{t}_{\mathrm{k}, \mathrm{j}} \leq \gamma^{-2} \mathrm{I}_{\mathrm{th}, \mathrm{j}}
\end{gathered}
$$

Where $\mathrm{g}_{\mathrm{k}, \mathrm{i}}$ and $\mathrm{t}_{\mathrm{k}, \mathrm{j}}$ are the diagonal coeffients of matrices $\mathbf{G}_{\mathrm{i}}^{\mathrm{H}} \mathbf{G}_{\mathrm{i}}$ and $\mathbf{T}_{\mathrm{j}}^{\mathrm{H}} \mathbf{T}_{\mathrm{j}}$ correspondingly.

Now the Lagrangian operator will be:

$$
\begin{aligned}
& \mathrm{L}_{\mathrm{pp}}=-\sum_{\mathrm{k}=1}^{\mathrm{M}} \log \left(1+\sigma_{\mathrm{M}, \mathrm{k}} \mathrm{x}_{\mathrm{k}}\right)-\sum_{\mathrm{k}=1}^{\mathrm{M}} \eta_{\mathrm{k}} \mathrm{x}_{\mathrm{k}} \\
& +\mathrm{v}_{1}\left(\sum_{\mathrm{k}=1}^{\mathrm{M}} \mathrm{x}_{\mathrm{k}}-\gamma^{-2} \mathrm{P}_{\mathrm{T}}\right)+v_{2} \sum_{\mathrm{j}=1}^{\mathrm{P}}\left(\sum_{\mathrm{i}=\mathrm{k}}^{\mathrm{M}} \mathrm{x}_{\mathrm{k}} \mathrm{t}_{\mathrm{k}, \mathrm{j}}-\gamma^{-2} \mathrm{I}_{\mathrm{th}, \mathrm{j}}\right)
\end{aligned}
$$

The following KTT conditions apply:

$$
\begin{gathered}
\frac{\sigma_{\mathrm{M}, \mathrm{k}}}{1+\sigma_{\mathrm{M}, \mathrm{k}} \mathrm{x}_{\mathrm{k}}}-\eta_{\mathrm{k}}+\mathrm{v}_{1}+\mathrm{v}_{2} \mathrm{t}_{\mathrm{k}, \mathrm{j}}=0 \\
\eta_{\mathrm{k}} \mathrm{x}_{\mathrm{k}}=0, \mathrm{k}=1, \ldots, \mathrm{M} \\
\mathrm{x}_{\mathrm{k}} \geq 0, \mathrm{k}=1, \ldots, \mathrm{M} \\
\sum_{\mathrm{k}=1}^{\mathrm{M}} \mathrm{x}_{\mathrm{k}} \leq \gamma^{-2} \mathrm{P}_{\mathrm{T}} \text { and } \sum_{\mathrm{j}=1 \mathrm{k}=1}^{\mathrm{P}} \sum_{\mathrm{k}}^{\mathrm{M}} \mathrm{x}_{\mathrm{k}} \mathrm{t}_{\mathrm{k}, \mathrm{j}} \leq \sum_{\mathrm{j}=1}^{\mathrm{P}} \gamma^{-2} \mathrm{I}_{\mathrm{th}, \mathrm{j}}
\end{gathered}
$$

Therefore,

$$
\mathrm{x}_{\mathrm{k}}=\max \left\{\left(\mathrm{v}_{1}+\mathrm{v}_{2} \sum_{\mathrm{j}=1}^{\mathrm{P}} \mathrm{t}_{\mathrm{k}, \mathrm{j}}-\eta_{\mathrm{k}}\right)^{-1}-\sigma_{\mathrm{M}, \mathrm{k}}^{-1}, 0\right\}
$$

Substitute (18) to (17), I have:

$$
\mathrm{v}_{2} \geq \frac{\left[\mathrm{M}-\left(\gamma^{-2} \mathrm{P}_{\mathrm{T}}+\mathrm{a}_{1}-1\right) \mathrm{v}_{1}\right]}{\left(\gamma^{-2} \mathrm{I}_{\mathrm{th}, \mathrm{j}}+\mathrm{a}_{2}-1\right)}
$$

Here $\mathrm{a}_{1}=1+\sum_{\mathrm{i}=1}^{\mathrm{M}} \sigma_{\mathrm{M}, \mathrm{i}}^{-1}$ and $\mathrm{a}_{2}=1+\sum_{\mathrm{i}=1}^{\mathrm{M}} \sum_{\mathrm{j}=1}^{\mathrm{P}} \mathrm{t}_{\mathrm{k}, \mathrm{j}} \sigma_{\mathrm{M}, \mathrm{i}}^{-1}$.

Make it even more compact:

$$
\mathrm{v}_{2} \geq \frac{\mathrm{M}-\mathrm{A} \mathrm{v}_{1}}{\mathrm{~B}}
$$

with $A=\gamma^{-2} P_{T}+a_{1}-1$ and $B=\sum_{j=1}^{P} \gamma^{-2} I_{t h, j}+a_{2}-1$.

Substitute (20) to (18), I have:

$$
\mathrm{x}_{\mathrm{k}} \geq \mathrm{B}\left(\left(\mathrm{B}-\mathrm{A} \sum_{\mathrm{j}=1}^{\mathrm{P}} \mathrm{t}_{\mathrm{k}, \mathrm{j}}\right) \mathrm{v}_{1}+\mathrm{M} \sum_{\mathrm{j}=1}^{\mathrm{P}} \mathrm{t}_{\mathrm{k}, \mathrm{j}}\right)^{-1}-\sigma_{\mathrm{M}, \mathrm{k}}^{-1}
$$

$$
\mathrm{L}_{\mathrm{pp}}=-\sum_{\mathrm{k}=1}^{\mathrm{M}} \log \frac{\mathrm{B} \sigma_{\mathrm{M}, \mathrm{k}}}{\left(\mathrm{B}-\mathrm{A} \sum_{\mathrm{j}=1}^{\mathrm{P}} \mathrm{t}_{\mathrm{k}, \mathrm{j}}\right) \mathrm{v}_{1}+\mathrm{M} \sum_{\mathrm{j}=1}^{\mathrm{P}} \mathrm{t}_{\mathrm{k}, \mathrm{j}}}
$$

First order derivative:

$$
L_{p p}^{\prime}=-\sum_{k=1}^{M} \log \frac{B-A \sum_{j=1}^{P} t_{k, j}}{\left(B-A \sum_{j=1}^{P} t_{k, j}\right) v_{1}+M \sum_{j=1}^{P} t_{k, j}}
$$

Second derivative:

$$
L_{p p}^{\prime \prime}=-\sum_{k=1}^{M} \log \frac{\left(B-A \sum_{j=1}^{P} t_{k, j}\right)^{2}}{\left[\left(B-A \sum_{j=1}^{P} t_{k, j}\right) v_{1}+M \sum_{j=1}^{P} t_{k, j}\right]^{2}}
$$

Based on the condition: $0<\mathrm{x}_{\mathrm{k}} \leq 1$ and $0<\mathrm{x}_{\mathrm{k}} \mathrm{t}_{\mathrm{k}, \mathrm{j}} \leq 1$, the upper and lower limits are given as (25), (26).

I have maximal lower limit and minimum upper limit:

$$
\begin{aligned}
& \mathrm{v}_{\mathrm{lb}}=\max _{\mathrm{k}=\mathrm{l}: \mathrm{M}}\left\{\mathrm{v}_{\mathrm{k}}^{\mathrm{lb}}\right\} \\
& \mathrm{v}_{\mathrm{ub}}=\min _{\mathrm{k}=\mathrm{l}: \mathrm{M}}\left\{\mathrm{v}_{\mathrm{k}}^{\mathrm{ub}}\right\}
\end{aligned}
$$

There are some cases to find optimal $\mathrm{v}_{1}$ :

Firstly $\mathrm{v}_{\mathrm{lb}}=\mathrm{v}_{\mathrm{ub}}$, then $\mathrm{v}_{1}=\mathrm{v}_{\mathrm{lb}}=\mathrm{v}_{\mathrm{ub}}$ Secondly, $\mathrm{v}_{\mathrm{lb}}<\mathrm{v}_{\mathrm{ub}}$ and $\mathrm{L}_{\mathrm{pp}}^{\prime}\left(\mathrm{v}_{\mathrm{ub}}\right) \geq 0$, then $\mathrm{v}_{1}=\mathrm{v}_{\mathrm{ub}}$

Thirdly, $\mathrm{v}_{\mathrm{lb}}<\mathrm{v}_{\mathrm{ub}}$ and $\mathrm{L}_{\mathrm{pp}}^{\prime}\left(\mathrm{v}_{\mathrm{lb}}\right) \leq 0$, then $\mathrm{v}_{1}=\mathrm{v}_{\mathrm{lb}}$ Fourthly, $\mathrm{v}_{\mathrm{lb}}<\mathrm{v}_{\mathrm{ub}}$ and $\mathrm{L}_{\mathrm{pp}}^{\prime}\left(\mathrm{v}_{\mathrm{lb}}\right)>0, \mathrm{~L}_{\mathrm{pp}}^{\prime}\left(\mathrm{v}_{\mathrm{ub}}\right)<0$, then $\mathrm{v}_{1}$ is found to satisfy $\mathrm{L}_{\mathrm{pp}}^{\prime}\left(\mathrm{v}_{1}\right)=0$.

Fifthly, $\mathrm{v}_{\mathrm{lb}}>\mathrm{v}_{\mathrm{ub}}$, the index $\mathrm{k}$ is gradually removed $\mathrm{k}=\mathrm{M}: 1$ so that $\mathrm{v}_{\mathrm{lb}}<\mathrm{v}_{\mathrm{ub}}$.

\section{SIMULATION}

I will compare two different algorithms to figure out which channel capacity is more significant than the other. With the assumptions $\mathrm{EH}=3$ (number of ERs), $\mathrm{M}=3$ (number of SUs), $\mathrm{G}=2$ (number of antennas of $\mathrm{ST}$ ), $\mathrm{NG}=3$ (number of creating precoder ), $\mathrm{F}$, and $\mathrm{H}$ are said to be random matrices of size $\mathrm{G} \times($ E.M.NG) and (E.M.NG) $\times \mathrm{H}$. In addition, I have assumed noise values through $\mathrm{K}=2$ (number of antennas for each $\mathrm{PU}$ ), $\mathrm{P}=3$ (number of $\mathrm{PUs}$ ). Ith is the noise vector of size $1 \times \mathrm{P}, \mathrm{EHa}=2$ (number of antennas for each $\mathrm{ER}$ ), $\mathrm{T}$

The Lagrange operator now becomes: 


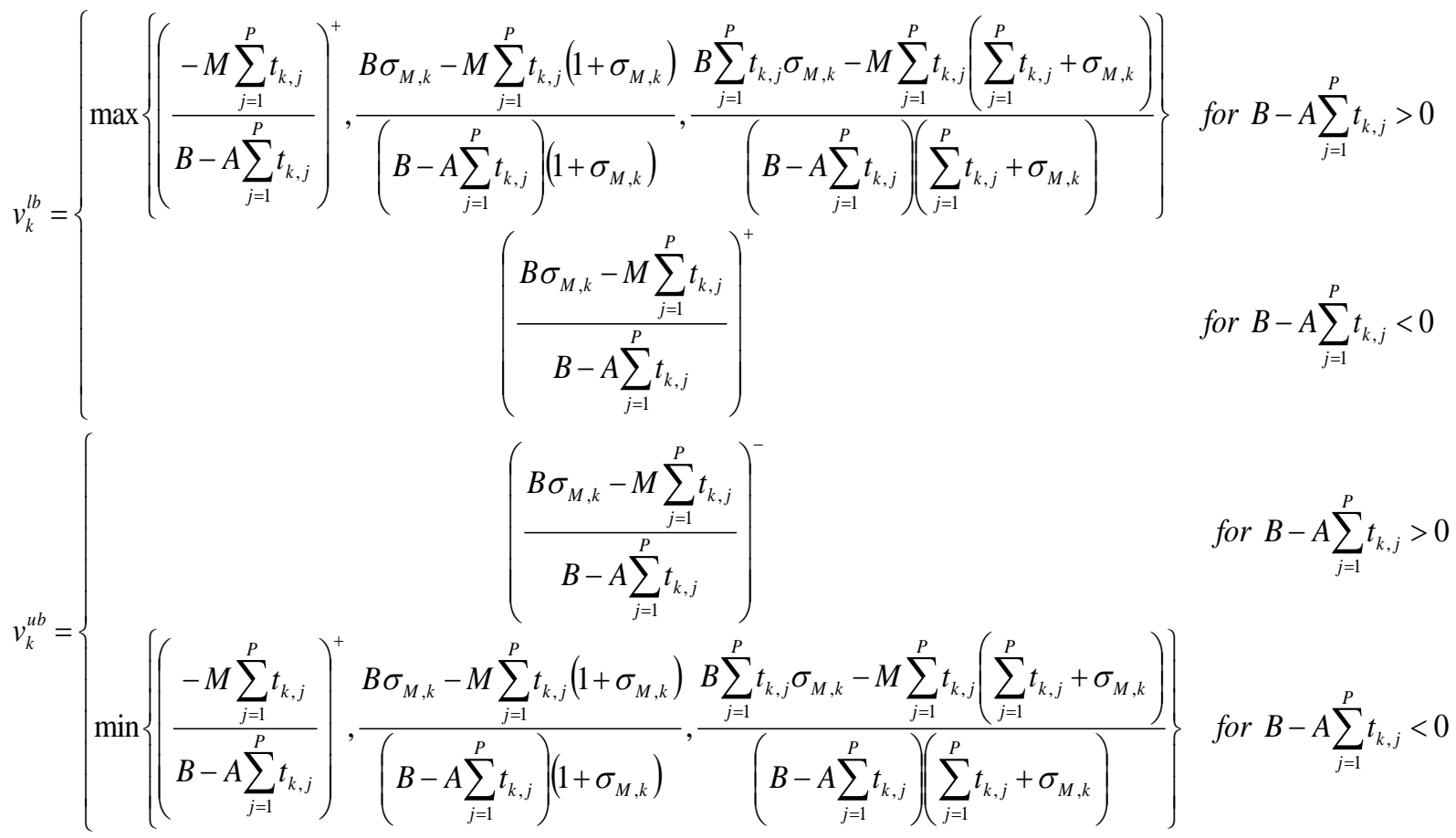

has size $(\mathrm{KP}) \times \mathrm{G}, \mathrm{u}$ is a random data vector of size $1 \times(\mathrm{EH}+\mathrm{P})$ and the channel matrix from ST to all ERs GEHhas size $(\mathrm{EHa}, \mathrm{EH}) \times \mathrm{G}$. When I compare the WMMSE algorithm (red line), using the MSR, higher capacity is achieved by the latter with the proposed method. (blue line), illustrated in Fig. 2 and Table. 1.

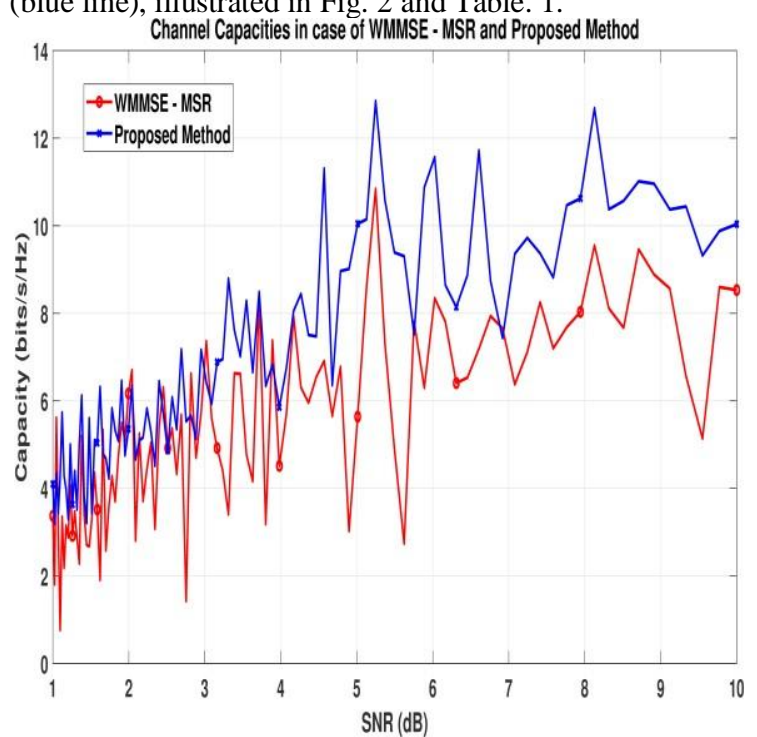

Fig.2: Capacities vs SNR in case of WMMSE - MSR and Proposed method

Table 1. Capacities vs SNR for WMMSE- MSR and Proposed method

\begin{tabular}{|c|c|c|c|c|c|}
\hline SNR (dB) & $\mathbf{1}$ & $\mathbf{3}$ & $\mathbf{5}$ & $\mathbf{7}$ & $\mathbf{9}$ \\
\hline $\begin{array}{c}\text { WMMSE- } \\
\text { MSR }\end{array}$ & 3.37 & 6.17 & 4.92 & 5.64 & 8.03 \\
\hline $\begin{array}{c}\text { Proposed } \\
\text { method }\end{array}$ & 4.1 & 5.36 & 6.89 & 10.04 & 10.62 \\
\hline
\end{tabular}

I will compare more this method with two other methods, HMR and PF. The improved method still gives higher capacity than HMR and PF methods, described in Fig. 3 and Table 2.

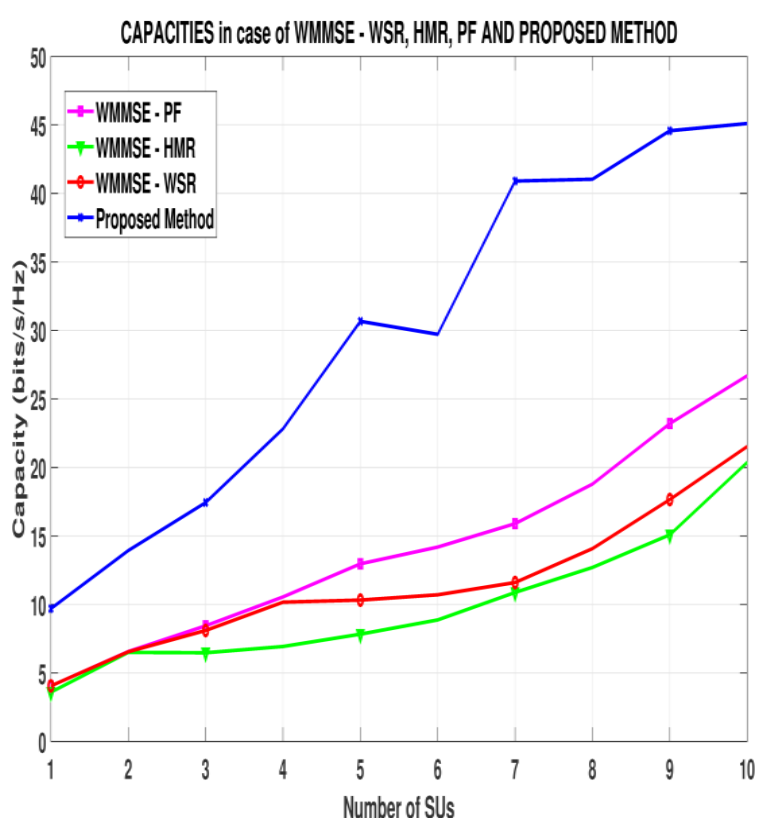

Fig.3: Capacities vs SNR in case of WMMSE - MSR, HMR, PF and Proposed method

Table 2. Capacities vs SNR for WMMSE- MSR, HMR, $\mathrm{PF}$ and Proposed method

\begin{tabular}{|c|c|c|c|c|c|}
\hline SNR (dB) & $\mathbf{1}$ & $\mathbf{3}$ & $\mathbf{5}$ & $\mathbf{7}$ & $\mathbf{9}$ \\
\hline PF & 5.1 & 6.24 & 7.15 & 8.17 & 9.29 \\
\hline HMR & 4.2 & 5.25 & 6.11 & 7.1 & 8.21 \\
\hline WSR & 1.74 & 2.43 & 3.09 & 4.57 & 5.99 \\
\hline $\begin{array}{c}\text { Proposed } \\
\text { method }\end{array}$ & 6.42 & 8.62 & 10.22 & 11.9 & 13.63 \\
\hline
\end{tabular}


If I increase the number of SUs among the three methods, the proposed method still makes the capacity higher, as shown in Fig. 4 and Table 3.

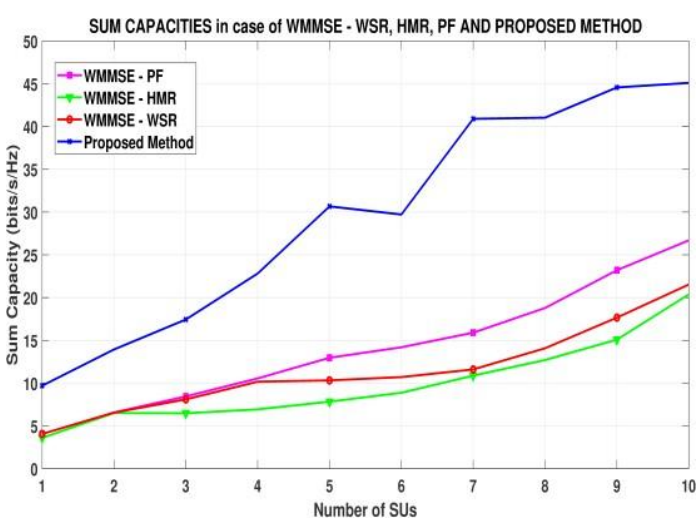

Fig.4: Capacities vs number of SUs in case of WMMSE MSR, HMR, PF and Proposed method

Table 3. Capacities vs number of SUs for WMMSEMSR, HMR, PF and Proposed method

\begin{tabular}{|c|c|c|c|c|c|}
\hline $\begin{array}{c}\text { SNR } \\
(\mathbf{d B})\end{array}$ & $\mathbf{1}$ & $\mathbf{3}$ & $\mathbf{5}$ & $\mathbf{7}$ & $\mathbf{9}$ \\
\hline PF & 4.05 & 8.45 & 12.97 & 15.9 & 23.2 \\
\hline HMR & 3.6 & 6.48 & 7.83 & 10.88 & 15.09 \\
\hline WSR & 4.06 & 8.1 & 10.32 & 11.6 & 17.66 \\
\hline $\begin{array}{c}\text { Proposed } \\
\text { method }\end{array}$ & 9.7 & 17.44 & 30.66 & 40.9 & 44.57 \\
\hline
\end{tabular}

Moving on, I increase the number of ERs and see the channel capacity using the proposed method is higher than the methods of WMMSE, seen from Fig. 5 and Table 4.

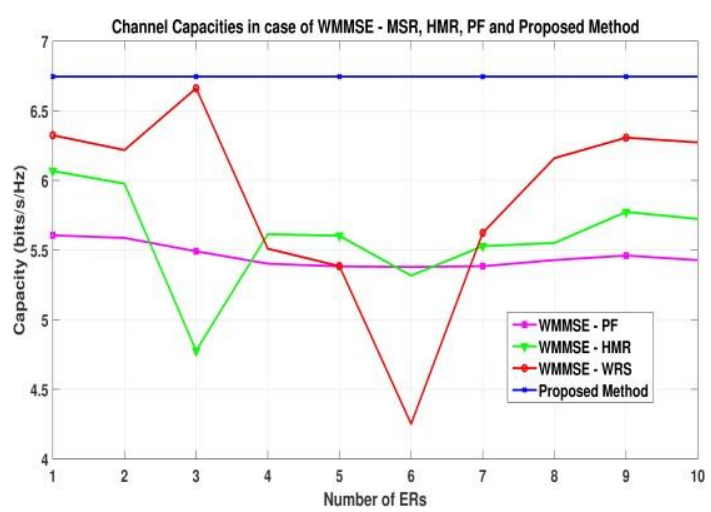

Fig.5: Capacities vs number of ERs in case of WMMSE MSR, HMR, PF and Proposed method

Table 4. Capacities vs number of ERs for WMMSEMSR, HMR, PF and Proposed method

\begin{tabular}{|c|c|c|c|c|c|}
\hline $\begin{array}{c}\text { SNR } \\
(\mathbf{d B})\end{array}$ & $\mathbf{1}$ & $\mathbf{3}$ & $\mathbf{5}$ & $\mathbf{7}$ & $\mathbf{9}$ \\
\hline PF & 5.61 & 5.49 & 5.38 & 5.38 & 5.46 \\
\hline HMR & 6.07 & 4.78 & 5.6 & 5.53 & 5.77 \\
\hline WSR & 6.32 & 6.66 & 5.38 & 5.62 & 6.31 \\
\hline $\begin{array}{c}\text { Proposed } \\
\text { method }\end{array}$ & 6.75 & 6.75 & 6.75 & 6.75 & 6.75 \\
\hline
\end{tabular}

When I increase the number of PUs, then get Fig. 6 and Table 5, where the capacity is the same in three cases, MSR, $\mathrm{HMR}$, and PF, and is lower than the proposed method.

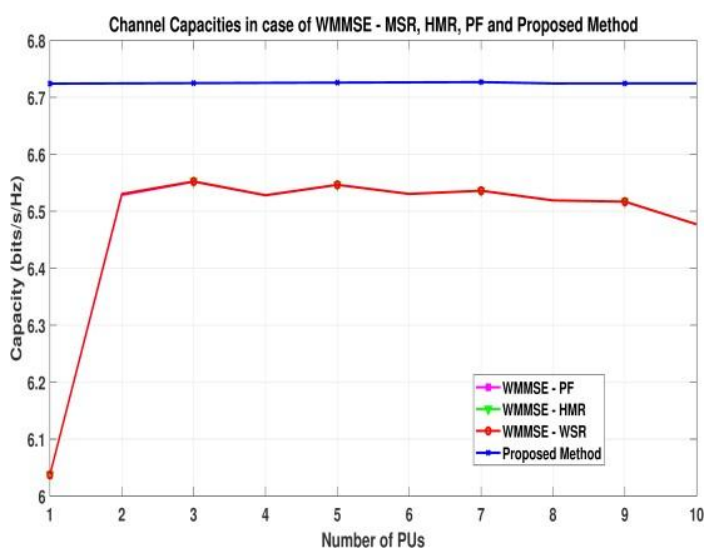

Fig.6: Capacities vs number of PUs in case of WMMSE MSR, HMR, PF and Proposed method

Table 5. Capacities vs number of PUs for WMMSEMSR, HMR, PF and Proposed method

\begin{tabular}{|c|c|c|c|c|c|}
\hline $\begin{array}{c}\text { SNR } \\
\text { (dB) }\end{array}$ & $\mathbf{1}$ & $\mathbf{3}$ & $\mathbf{5}$ & $\mathbf{7}$ & $\mathbf{9}$ \\
\hline $\begin{array}{c}\text { PF, HMR, } \\
\text { WSR }\end{array}$ & 6.04 & 6.55 & 6.55 & 6.54 & 6.52 \\
\hline $\begin{array}{c}\text { Proposed } \\
\text { method }\end{array}$ & 6.72 & 6.72 & 6.73 & 6.73 & 6.72 \\
\hline
\end{tabular}

In addition, I also use additional noise parameters from ST to PUs to be determined; I have Fig.7 and Table. 6, in which the proposed method still provides a higher channel capacity than other methods: MRS, HMR and PF.

Figures 2 and 3 and Tables 1 and 2, respectively, describe the channel capacity vs. SNR received by each SU using the new method, which is more significant than other methods such as WSR, HMR, and PF in the same determined environment. This environment has the same fading matrices between ST and SUs, PUs and ERs, number of PUs, SUs, and ERs. In addition, the author changes these numbers to see the capacity of the ST to the SUs. The author has changed the number of SUs (Figure 4, Table 3), the number of ERs (Figure 5, Table 4), the number of PUs (Figure 6, Table 5), or change the noise value from ST to PUs (Figure 7, Table 6 ). The results show that the capacity according to the proposed method is still more significant than that of the traditional methods such as WSR, HMR, and PF.

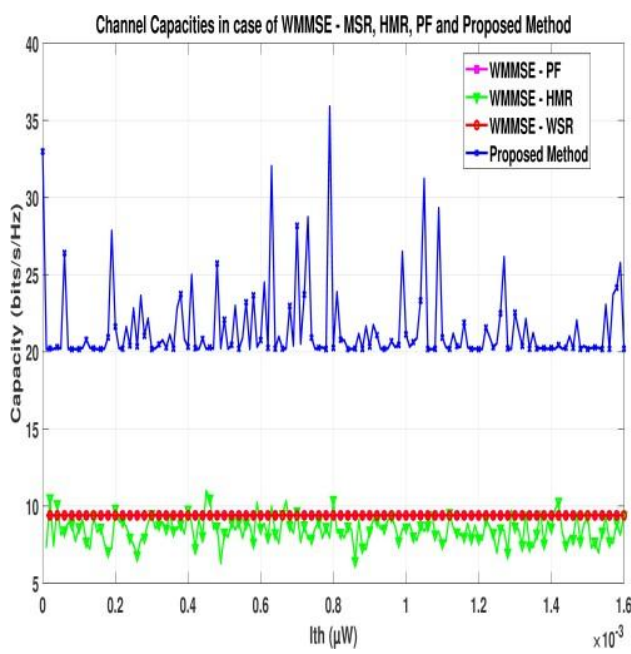

Fig.7: Capacities vs $I_{\text {th }}$ in case of WMMSE - MSR, HMR, PF and Proposed method 
Table 6. Capacities vs $I_{\text {th }}$ for WMMSE- MSR, HMR, PF and Proposed method

\begin{tabular}{|c|c|c|c|c|c|}
\hline $\begin{array}{c}\text { SNR } \\
(\mathbf{d B})\end{array}$ & $\mathbf{0 . 3}$ & $\mathbf{0 . 6}$ & $\mathbf{0 . 9}$ & $\mathbf{1 . 2}$ & $\mathbf{1 . 5}$ \\
\hline PF & 9.41 & 9.41 & 9.41 & 9.41 & 9.41 \\
\hline HMR & 9.47 & 8.58 & 84.8 & 79.00 & 9.32 \\
\hline WSR & 9.39 & 9.39 & 9.39 & 9.39 & 9.39 \\
\hline $\begin{array}{c}\text { Proposed } \\
\text { method }\end{array}$ & 20.18 & 20.76 & 20.33 & 20.17 & 20.18 \\
\hline
\end{tabular}

\section{CONCLUSION}

Cognitive radio systems (CRSs) are considered a system of interest due to their ability to enhance spectrum reuse of primary transmitters for secondary transmitters when using the spectrum sensing technique. However, some constraints include the effect of secondary transmitters on primary users or the power allocated to the energy harvesting devices. Several methods have been given, of which the combined method of error reduction and capacity increase WMMSE is considered superior. Attached are several methods of lighted sum rate (WSR), harmonic mean rate (HMR), or proportional fairness (PF). An proposed method is given when using the SVD method for eigenvectors and using the generalised Lagrange method to optimize optimal power distribution values for eigenvectors. This method gives a higher capacity than the three methods mentioned above

\section{ACKNOWLEDGMENTS}

I thank the University of Transport and Communications, Hanoi, Vietnam for supporting this paper.

\section{REFERENCES}

[1] Thomas, R. W., Dasilva, L. A., Mackenzie, "A. B. Cognitive networks", In First IEEE International Symposium on New Frontiers in Dynamic Spectrum Access Networks. Baltimore, MD (USA), 2005. DOI: 10.1109/DYSPAN.2005.1542652

[2] Umar, R., Sheikh, A. U. H., "Cognitive radio oriented wireless networks: challenges and solutions. International Conference on Multimedia Computing and Systems", Tangiers (Morocco), 2012. DOI: 10.1109/ICMCS.2012.6320105

[3] Nguyen, V. T., Villain, F., Guillou, Y. L., "Cognitive radio RF: overview and challenges. Hindawi Publishing Corporation", $2012, \quad$ vol.
DOI:10.1155/2012/716476

[4] Chatterjee, S., Roy, J. S., Bhattacharya, P. P., "Spectrum sensing techniques for cognitive radio - a survey", International Journal of Applied Engineering Research, 2015, vol.10, no. 7, p. 16665-16684

[5] Kusaladharma, S., Tellambura, C., "An Overview of Cognitive Radio Networks, Wiley Encyclopedia of Electrical and Electronics Engineering", John Wiley \& Sons, Inc, 2017, p.1-17. DOI: 10.1002/047134608x.w8355.

[6] Zhang, T., Huo, Y, Zhao, J. "Cooperative precoding design for cognitive wireless energy transfer networks", IEICE Communications Express, 2018, vol.7, no.10, p.358-363. DOI: 10.1587/comex.2018XBL0089.

[7] Song, C., Lee, H., Lee, K. J., "Optimal Precoder Designs for Sum-Utility Maximization in SWIPTEnabled Multi-User MIMO Cognitive Radio Networks", EEE Systems Journal, 2019, vol. 13, issue. 3, p. 2332 2343. DOI: $10.1109 /$ JSYST.2018.2875762

[8] Al-Ali, M. H., Ho, K. C., "Precoding for mimo channels in cognitive radio networks with csi uncertainties and for mimo compound capacity", IEEE Transactions on Signal Processing, 2017, vol. 65, issue. 15, p. 3976 3989. DOI: 10.1109/TSP.2017.2701320

[9] Jiang, L., Tian, H., Qin, C., Gjessing, S., Zhang, Y., "Secure beamforming in wireless-powered cooperative cognitive radio networks", IEEE Communications Letters, 2016. vol.20, issue.3, p. 522-525, DOI: 10.1109/LCOMM.2016.2514353

[10] Maurya, S., Bansal, M., "Design of energy-efficient precoders in MIMO cognitive two-way relay network", Wireless Networks, 2020, vol. 26, issue. 1, pp. 293-305. DOI: $10.1007 / \mathrm{s} 11276-018-1813-1$

[11] Mou, W., Yang, W., Huang, Y., Xu, X., Cai, Y., Wang, $\mathrm{K}$., "On the security of cooperative cognitive radio networks with distributed beamforming", EURASIP Journal on Wireless Communications and Networking, 2017, vol.144. DOI 10.1186/s13638-017-0923-2

[12] Zhu, J., "Security-reliability trade-off for cognitive radio networks in the presence of eavesdropping attack", EURASIP Journal on Advances in Signal Processing, 2013, vol. 169. DOI: 10.1186/1687-6180-2013-169 